\title{
SCIENTIFIC REPORTS

\section{OPEN Melatonin controls microbiota in colitis by goblet cell differentiation and antimicrobial peptide production through Toll-like receptor 4 signalling}

Seung Won Kim ${ }^{1,2,3}$, Soochan Kim ${ }^{1,3}$, Mijeong Son ${ }^{1,2,3}$, Jae Hee Cheon ${ }^{1,2,3}$ \& Young Sook Park ${ }^{4 *}$

Microbial dysbiosis has long been postulated to be associated with the pathogenesis of inflammatory bowel disease (IBD). Although evidence supporting the anti-colitic effects of melatonin have been accumulating, it is not clear how melatonin affects the microbiota. Herein, we investigated the effects of melatonin on the microbiome in colitis and identified involvement of Toll-like receptor (TLR) 4 signalling in the effects. Melatonin improved dextran sulfate sodium (DSS)-induced colitis and reverted microbial dysbiosis in wild-type (WT) mice but not in TLR4 knockout (KO) mice. Induction of goblet cells was observed with melatonin administration, which was accompanied by suppression of $/ l 1 b$ and $l 117 a$ and induction of melatonin receptor and Reg $3 \beta$, an antimicrobial peptide (AMP) against Gram-negative bacteria. In vitro, melatonin treatment of HT-29 intestinal epithelial cells promotes mucin and wound healing and inhibits growth of Escherichia coli. Herein, we showed that melatonin significantly increases goblet cells, Reg3 $\beta$, and the ratio of Firmicutes to Bacteriodetes by suppressing Gram-negative bacteria through TLR4 signalling. Our study suggests that sensing of bacteria through TLR4 and regulation of bacteria through altered goblet cells and AMPs is involved in the anti-colitic effects of melatonin. Melatonin may have use in therapeutics for IBD.

Inflammatory bowel disease (IBD) is a chronic relapsing disorder of the gastrointestinal (GI) $\operatorname{tract}^{1}$ that results in chronic diarrhoea, abdominal pain, weight loss, and altered gastrointestinal motility ${ }^{2}$. The pathogenesis of IBD is considered to be multidimensional and multifactorial, involving genetic and environmental factors. The microbiota (microbial community assemblage) plays a critical role in the GI tract and is involved in nutrient digestion, vitamin synthesis, and promotion of mucosal immune tolerance. Perturbation of the intestinal microbiota composition and inflammatory response can aggravate intestinal barrier dysfunction and is a risk factor for IBD, although the mechanisms of IBD pathogenesis remain unclear. While various pharmaceutical treatments are available for $\mathrm{IBD}^{3}$, some therapeutic agents have a higher risk of toxicity and infectious complications ${ }^{4}$. Recently, microbiota control using faecal microbiota transplantation, probiotics, and prebiotics has been proposed as a new therapeutic option ${ }^{5}$.

Melatonin (N-acetyl-5-methoxy ${ }^{5}$ tryptamine) is a pineal gland hormone that regulates other hormones, circadian rhythm, and oxidative stress ${ }^{6}$. Recent experiments have shown that melatonin also acts as an immune system modulator $^{7}$ and ameliorates DSS colitis in mice ${ }^{8,9}$. Numerous studies have indicated that melatonin affects the permeability, motility, and barrier function of the intestine $\mathrm{e}^{10,11}$. Melatonin treatment can improve GI tract diseases, such as irritable bowel syndrome ${ }^{12}, \mathrm{IBD}^{13}$, and necrotizing enterocolitis ${ }^{14}$, suggesting it has a critical role in the physiologic function of the GI tract. Interestingly, melatonin accumulates in the GI tract independent of production from the pineal gland. The concentration of melatonin in the GI tract is 400 -fold greater than that in the pineal gland $^{15}$. The GI tract is also the largest source of enzymes for melatonin production ${ }^{16}$, and melatonin receptors are

${ }^{1}$ Department of Internal Medicine and Institute of Gastroenterology, Yonsei University College of Medicine, Seoul, Korea. ${ }^{2}$ Brain Korea 21 PLUS Project for Medical Science, Yonsei University College of Medicine, Seoul, Korea. ${ }^{3}$ Severance Biomedical Science Institute, Yonsei University College of Medicine, Seoul, Korea. ${ }^{4}$ Department of Internal Medicine, Eulji Hospital, Eulji University School of Medicine, Seoul, Korea. *email: pys1109@eulji.ac.kr 
highly expressed in the GI tract, suggesting that melatonin affects the immune response and the microbiota. Recent studies reported that melatonin increases the ratio of Firmicutes to Bacteroidetes and Akkermania ${ }^{10}$ and suppresses pathogenic bacteria ${ }^{17}$ in the intestine. Despite this knowledge, the pivotal signal pathway for the anti-colitic effect of melatonin and the exact mechanism for control of the intestinal microbiota remains unknown.

We previously reported that melatonin improves sleep deprivation-induced colitis in mice ${ }^{13}$. In this study, we investigate the involvement of Toll-like receptor (TLR) 4 signal in the action of melatonin. TLR4 is an important TLR that recognizes pathogen-associated molecular patterns ${ }^{18}$, especially Gram-negative bacteria, and controls intestinal epithelial cells and the mucosal barrier. We also explored the effects on microbiota dysbiosis and antimicrobial peptide (AMP) regulation of melatonin in colitis.

\section{Results}

Intraperitoneal administration of melatonin ameliorates DSS-induced colitis through TLR4 signalling. TLR responses in intestinal epithelium by commensal bacteria play a significant role in the innate immunity, colonic homeostasis, and tolerance that link luminal bacteria and intestinal inflammation ${ }^{19}$. Among these TLRs, TLR4 recognize lipopolysaccharide (LPS) in particular and is primarily involved in control of Gramnegative bacteria; an association between genetic polymorphisms of TLR4 and IBD have been reported, although some controversy remains ${ }^{19}$. TLR4 knockout (KO) mice are highly susceptible to Gram-negative bacteria ${ }^{20,21}$. Thus, to explore the involvement of TLR4 signalling in the anti-colitic effects of melatonin in vivo, colitis was induced in 8 -week-old wild type (WT) and TLR4 KO mice by administration of 2.5\% dextran sulfate sodium (DSS) in drinking water and intraperitoneal (i.p.) treatment with melatonin (Mel) or vehicle (Veh). Body weight changes were monitored daily. DSS-treatment for 10 days significantly increased body weight loss in the vehicle-treated group of both wild type and TLR4 KO mice. Although there were no significant differences in body weights between DSS + Veh and DSS + Mel groups (Fig. S1a,b), melatonin improved the disease activity index (DAI) (Fig. 1a,b) and attenuated shortening of the colon (Fig. 1c,d) only in wild type mice but not in TLR4 KO mice.

Defects of TLR4 signalling abolishes induction of goblet cells and anti-inflammatory response caused by melatonin. Colons of the DSS-treated group indicated severe inflammation with inflammatory cell infiltration and microscopic damage including transmural injury, crypt and epithelium loss, and goblet cell loss. In contrast, the melatonin-treated group of wild type mice showed significant improvement in histopathologic features compared to the vehicle-treated group, while these effects were not observed in TLR4 KO mice (Fig. 1e-h). Of note, periodic acid-Schiff (PAS) staining indicated that the melatonin-treated control group displayed drastically increased goblet cells in colons, whereas TLR4 KO mice showed a trend toward decreased goblet cells compared to WT mice (Fig. 1e,f,i,j).

Colitis is characterized by expression of both pro-inflammatory cytokines, including tumour necrosis factor-alpha (Tnfa), interleukin 1- $\beta$ (Illb), and interleukin $17 \mathrm{~A}$ (Il17a), and anti-inflammatory cytokines, including interleukin 10 (Il10) and interleukin 22 (Il22). Gene expression of these cytokines in colons was investigated. Real-time quantitative reverse-transcription polymerase chain reaction (qRT-PCR) analysis indicated that melatonin significantly suppressed induction of Ill $b$ and $I l 17 a$ in WT mice, which was not true in TLR4 KO mice (Fig. 2a). Of note, TLR4 KO mice showed a trend of increased Tnfa, Illb, and Illi $a$ but reduced Il10 compared to WT mice (Fig. 2a,b). Taken together, these results indicate that melatonin modulates goblet cell differentiation and expression of inflammatory cytokines via TLR4 signalling.

Melatonin alter microbiota composition and corrects gut dysbiosis. There are two different receptors for melatonin, type 1 A (MT1) and type 1B (MT2), which are encoded by Mtnr1 $a$ and Mtnr1b, respectively 22 and expressed in the intestine ${ }^{23-25}$. Expression of melatonin receptors (Mtnrla and Mtnr1b) was assessed to investigate changes in melatonin and TLR4 signalling. qRT-PCR showed significantly increased expression of Mtnrla and an increasing trend of $M t n r 1 b$ in colons of melatonin-administered mice compared to vehicle-treated groups when also treated with DSS (Fig. 2c). However, expression levels of Mtnrla showed a decreased trend in TLR4 KO mice compared to WT mice. Immunohistochemistry staining for MT1 in colon sections also supported these results (Fig. 2d-f). TLR4 is an important signal pathway in homeostasis between hosts and pathogens. The results indicate that melatonin signalling is linked to TLR4 signalling and affects the microbiota, and there is a critical modulator dependent on TLR4 signalling.

First, to observe changes in microbiota caused by melatonin, metagenome analysis was performed using faeces of WT and TLR4 KO mice. 16S rRNA gene high-throughput pyrosequencing revealed the overall microbiome composition (beta diversity) assessed using principal coordinate analysis (PCoA), which showed distinct differences in clustering between the microbiomes of vehicle- and melatonin-treated groups and between WT and TLR4 KO mice (Fig. 3a). In alpha-diversity analysis, the melatonin-administered group showed significantly increased richness of faecal microbiota assessed using Chao index (Fig. 3b), increasing trends in ACE and Shannon diversity index, and a decreased trend of Simpson index compared to DSS + Veh groups (Fig. S2a), indicating that melatonin increased richness and diversity of the microbiome. Of note, melatonin-treated groups showed significant suppression of Proteobacteria, a representative Gram-negative phylum that includes Salmonella and Escherichi coli (Fig. 3c), and increased Ruminococcaceae family strains (Fig. S2d), which represent butyrate-producing Gram-positive bacteria decreased in abundance in IBD fecal microbiota ${ }^{26,27}$. While the difference was not significant, melatonin-treated groups showed a decreasing trend in Bacteroidetes and an increasing trend of Firmicutes relative abundance compared to vehicle-treated groups (Fig. 3c) as previous studies ${ }^{17,28}$. A significantly decreased Firmicutes to Bacteroidetes ratio in DSS-treated groups was also shown in TLR4 KO mice, whereas a significantly decreased abundance of Proteobacteria was shown in DSS-treated groups of WT mice (Figs. 3c,d and S2b-f). 

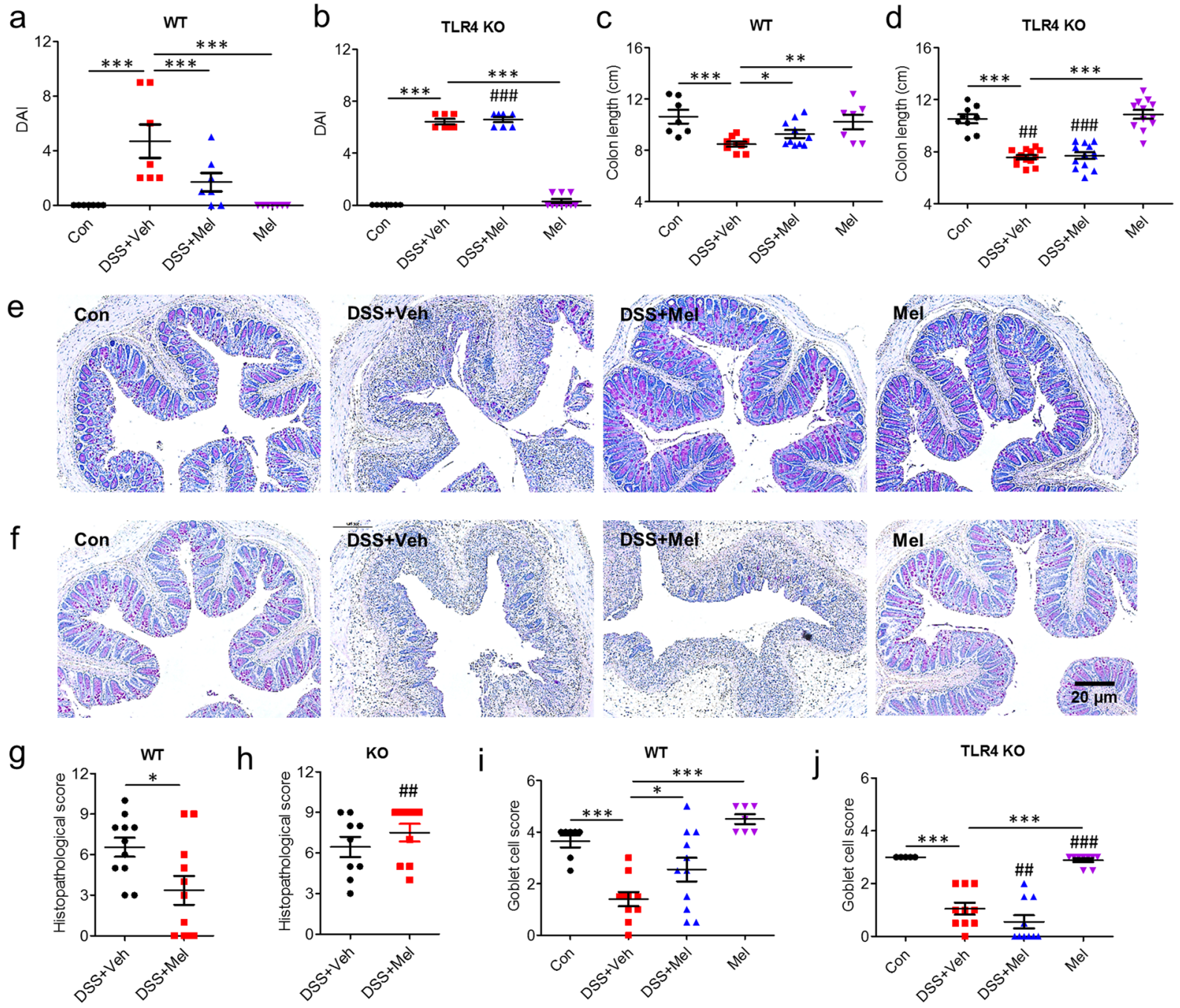

Figure 1. Intraperitoneal administration of melatonin ameliorates DSS-induced colitis through TLR4 signal pathway. Wild type (WT) and TLR4 knockout (TLR4 KO) mice were administered 2.5\% DSS in drinking water and treated i.p. with $0.25 \%$ EtOH/PBS (Veh) or melatonin (Mel, $10 \mathrm{mg} / \mathrm{kg} /$ day) from days 1 to 8 . (a,b) Disease activity index (DAI). (c,d) Colon length. (e-j) Histopathology of colon. (e,f) Representative image of periodic acid-Schiff (PAS)-stain. (g,h) Histopathologic score. $(\mathbf{i}, \mathbf{j})$ Goblet cell score. Data represent mean \pm S.E.M. $(\mathrm{n}=10)$. Statistical significance was assessed using one-way ANOVA followed by Dunnett post-test compared to DSS + Veh. $* P<0.05,{ }^{*} P<0.01, * * * P<0.005,{ }^{\# \#} P<0.01$ vs. TLR4 KO, ${ }^{\# \#} P<0.005$ vs. TLR4 KO. Con, untreated control.

Melatonin controls Gram-negative bacteria through induction of Reg3 $\beta$. Next, gene expression related to AMPs in the proximal colon was examined, including cathelin-related antimicrobial peptide (Cramp), regenerating islet-derived protein 3 beta $(R e g 3 b)$, defensin alpha 3 (Defa3), and defensin alpha 4 (Defa4), to explore the mechanism of microbiota regulation in the colon. Among these AMPs, only Reg $3 b$ expression was significantly increased in melatonin-administered groups compared to other groups, in which Reg3b expression was undetectable. Expression of other intestinal antimicrobial molecules was not affected (Fig. 4a,b). In concordance, protein levels assessed by immunostaining and western blot were also increased in the melatonin-administered groups (Fig. 4c-g). However, TLR4 KO mice showed reduced levels of inducible AMPs, such as Reg $3 \beta$ and defensins. This suggests that melatonin induces expression of AMPs, in particular Reg3 $\beta$, through TLR4 signalling.

Reg3 $\beta$ can be induced in goblet cells ${ }^{29}$, and melatonin promotes goblet cells and modulates cytokines. Moreover, since melatonin can modulate the function of macrophages ${ }^{30}$, HT-29 cells were treated with melatonin with or without THP-1 cells that were differentiated to macrophages by PMA stimulation and Alcian blue stain was performed to assess whether melatonin induced goblet cell differentiation in vitro. Melatonin treatment increased Alcian blue staining compared to the vehicle-treated group. However, there was no difference between HT-29 only (Fig. 5a) and co-culture with differentiated THP-1 cells (Fig. S4a). The increased Alcian blue stain was not detected when cells were treated with TLR4 inhibitor. Consistently, mRNA expression of MUC2, MTNR1A, and MTNR1B significantly increased after melatonin and LPS treatment, a finding that was eliminated when treated with TLR4 inhibitor (Fig. 5b,c). These results suggest that melatonin directly modulates goblet 
a

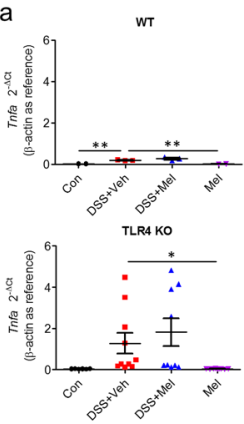

C
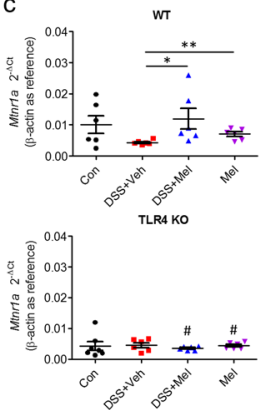
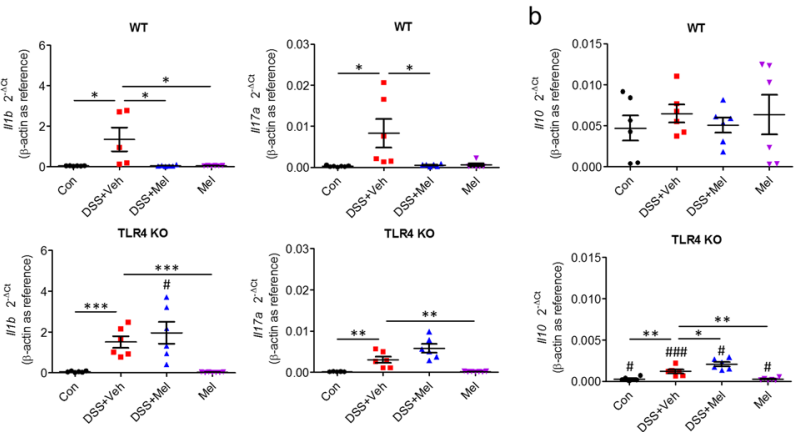

d
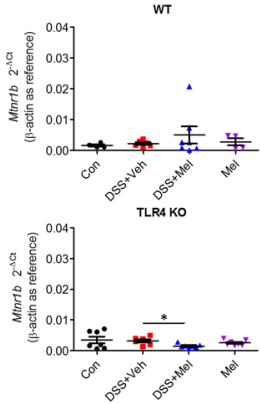
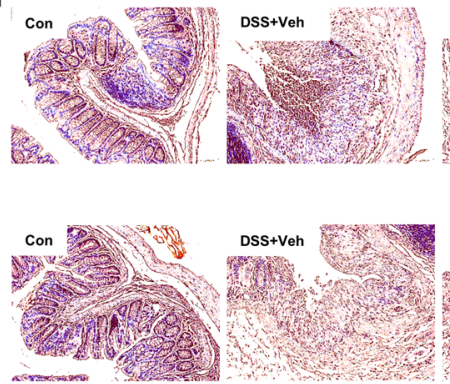
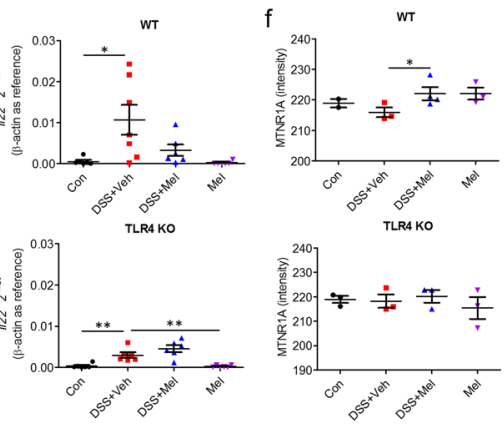

Figure 2. Melatonin modulates inflammatory cytokines and melatonin receptors in the colon through TLR4 signal pathway. (a-c) Gene expression in colon tissues. mRNA expression of pro-inflammatory cytokines (Tnfa, $I l 1 b, I l 17 a)(\mathbf{a})$, anti-inflammatory cytokines $(I l 10, I l 22)(\mathbf{b})$, and melatonin receptors $($ Mtnrla, Mtnr1b) (c) were evaluated by quantitative RT-PCR. (d-f) Protein levels of melatonin receptors in colon tissues from WT (d) and TLR4 KO (e) mice. (d,e) Representative image of immunohistochemistry for melatonin receptor 1 A (MTNR1A). (f) Densitometric analysis. Data represent mean \pm S.E.M. $(n=7)$. Statistical significance was assessed using Student's t-test. $* P<0.05, * * P<0.01, * * * P<0.005,{ }^{*} P<0.05$ vs. TLR4 KO, ${ }^{\# \#} P<0.005$ vs. TLR4 KO. Con, untreated control; Mel, melatonin-treated; TLR4 KO, TLR4 knockout mice; Veh, vehicle-treated; WT, wild-type mice.

cell differentiation, which requires a melatonin receptor and the TLR4 signal pathway. In addition, melatonin increased mRNA expression of a proinflammatory cytokine (TNFA) and an anti-inflammatory cytokine (IL10) as well as melatonin receptors (MTNR1A, MTNR1B) in differentiated THP-1 cells (Fig. 5e,f).

Upregulated expression of REG3G, CAMP, and DEFA3, the human orthologues of Reg3b, Cramp, and Defa3, in HT-29 cells was observed after melatonin only treatment or melatonin co-treatment with LPS (Fig. 5d), although induction of Cramp and Defa 3 was not induced by melatonin in vivo. Consistently, we observed upregulated expression of REG3G in differentiated THP-1 cells (Fig. 5g). To confirm that melatonin-treated cells suppress growth of Gram-negative bacteria in the pathogenesis of colitis, culture media of HT-29 cells or HT-29 cells co-cultured with differentiated THP-1 cells were treated with Escherichia coli, a representative Gram-negative bacterium. Dots of colonies and optical density of $E$. coli culture revealed that both melatonin-treated HT-29 cells and co-culture with THP-1 cells inhibited growth of $E$. coli, which was not affected by co-culture with THP-1 cells (Figs. 5h,i and S4b). These results suggest that melatonin suppresses Bacteroidetes through Reg3 $\beta$ induction.

Melatonin promotes wound healing in intestinal epithelial cells through TLR4 signalling. Previous studies have reported that TLR4 signalling promotes wound healing and induces AMPs in intestines $^{31}$. Moreover, expression of melatonin receptor is upregulated by melatonin through TLR4 signalling. To assess whether melatonin affects intestinal barrier regulation through wound repair, a wound healing assay was performed using scraped HT-29 cells with or without differentiated THP-1 cells. Melatonin promoted wound closure compared to the control, which was further increased by LPS stimulation. However, there was no significant difference between HT-29 cells only (Fig. 6a,b) and HT-29 cells co-cultured with THP-1 cells (Fig. S4c,d), suggesting that melatonin directly affects wound healing of IECs.

\section{Discussion}

Melatonin has versatile functions, including antioxidant, memory formation, and blood pressure reduction qualities ${ }^{32}$. Additionally, many previous studies showed that oral and rectal administration of melatonin suppresses DSS-induced colitis $^{33-35}$. Melatonin is abundant in the gut ${ }^{15}$, and pinealectomy has no influence on gut melatonin concentrations ${ }^{36}$. Although it is widely accepted that IBD involves perturbation of homeostasis between the gut microbiota and the host immune system ${ }^{37}$, the effects of melatonin on dysbiosis in IBD remain unknown. It is not yet clear how melatonin shapes biological functions, although recent studies have suggested that melatonin alters the microbiome of the GI tract ${ }^{10,17}$. Herein, we showed that melatonin i.p. administration in WT mice alleviates DSS-induced colitis. As with the previously reported effect of melatonin on DSS- or TNBS-induced colitis ${ }^{38,39}$, melatonin suppresses potent pro-inflammatory mediators in colitis such as $I l 1 b$ and $I l 17 a$, and controls microbiota in the intestine. 
a

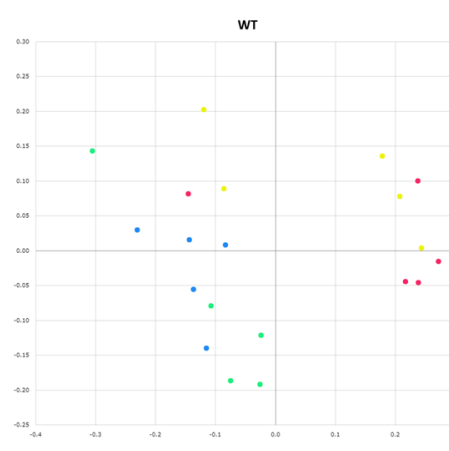

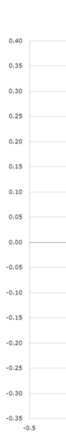

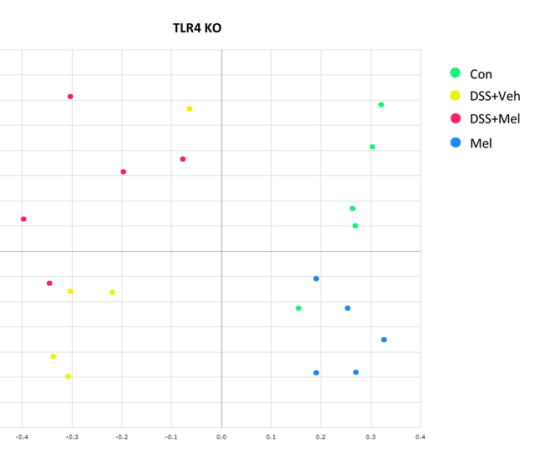

C
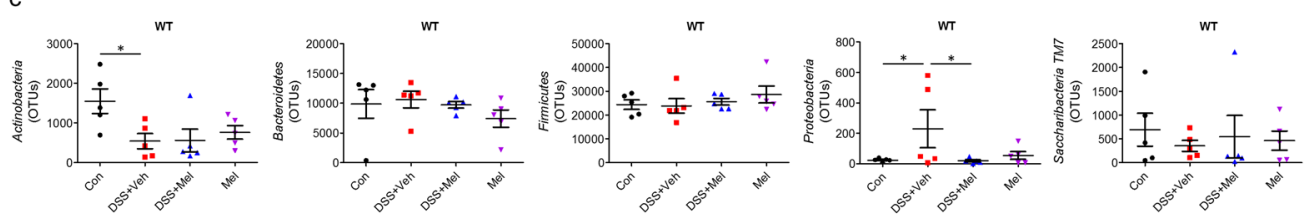

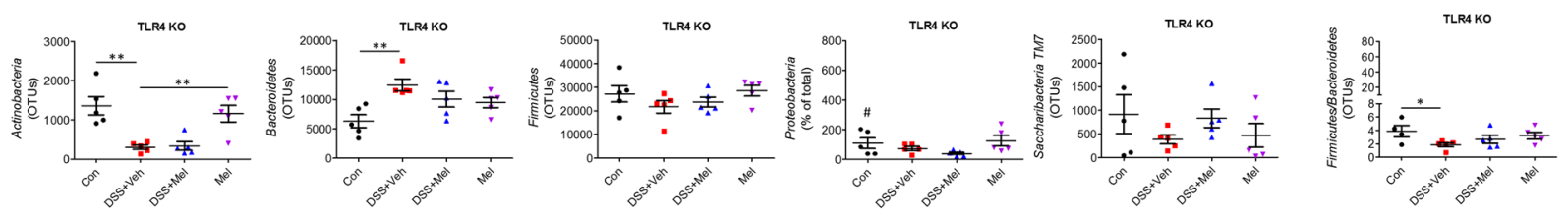

Figure 3. Melatonin suppresses dysbiosis of gut microbiome through TLR4 signal pathway. Fecal microbiome composition was generated using $16 \mathrm{~S}$ rRNA sequencing, and beta and alpha diversity and taxa were analysed. (a) Principal coordinate analysis (PCoA). (b) Chaol index. (c) Microbiota profiles in phylum level. (d) Firmicutes to Bacteroidetes ratio. Data represent mean \pm S.E.M. $(n=5)$. Statistical significance was assessed using Student's t-test (b,d) and one-way ANOVA followed by Dunnett post-test (c). $* P<0.05, * * P<0.01$. Con, untreated control; Mel, melatonin-treated; OUT, operational taxonomic unit; TLR4 KO, TLR4 knockout mice; Veh, vehicle-treated; WT, wild-type mice.

Consistent with our observation, many studies have reported that melatonin increases goblet cells in the intestine of vertebrates ${ }^{10,17,39,40}$. Goblet cells are important secretory IECs in colitis ${ }^{19}$ that produce mucin and AMPs. These products serve as the first lines of defence against pathogens by maintaining homeostasis of the mucus barrier and commensal bacteria and by segregating intestinal bacteria and the epithelium ${ }^{41}$. Considering that IECs interact with enteric bacteria, AMP production from IECs may affect the composition and abundance of the microbiota and play a role in protecting against bacterial invasion ${ }^{42}$, in addition to other types of cells such as $\mathrm{T}$ cell and innate lymphoid cells ${ }^{43}$. Although the route of administration was different from the previous study, we still identified indications that melatonin can reverse microbial dysbiosis in colitis and increase the Firmicutes to Bacteroidetes ratio. While melatonin has been reported to elevate the abundance of Firmicutes such as Lactobacillus and Akkermania, and reduce Bacteroidetes such as Prevotellaceae ${ }^{10,17}$, we did not observe any significant changes in these bacteria. This is likely due to different bacterial niches within the GI tract caused by the different sampling method. Given that mucin affects the composition of microbiota ${ }^{29}$ and some bacteria use glycan as a nutrient ${ }^{44,45}$, microbiota modulation by melatonin may be due to mucin regulation by goblet cell differentiation.

More importantly, host defences against Gram-negative bacteria such as Salmonella can be controlled by AMPs such as Reg3 $\beta$ and defensin alpha ${ }^{46-48}$. This may be another important mechanism of microbial control by melatonin. LPS in the cell walls of Gram-negative bacteria, pathogens commonly associated with IBD, induces an inflammatory response through TLR $4^{49}$. TLR4 is receptor for bacterial LPS and regulates LPS-induced inflammatory response. Gram-negative bacteria abundantly increase in TLR4 KO mice, and TLR4 induces differentiation of goblet cells and AMP expression, including Reg3 $\gamma$ and Reg3 $\beta^{42,50}$. We found Gram-negative bacteria such as Proteobacteria are elevated in the feces of TLR4 KI mice (Fig. 3c), while Firmicutes such as Ruminococcaceae are reduced in those of TLR4 KI mice (Fig. S2d). As in the previous study ${ }^{29,48}$, we identified that Reg $3 \beta$ can be induced in proximal colon tissue. The previous study reported that melatonin inhibited infection in an enterotoxigenic E. coli-infected model ${ }^{17}$. Our study is the first to show that melatonin increased AMPs, especially Reg3 $\beta$. Consistent with the antibacterial specificity of Reg3 $\beta$ for Gram-negative bacteria ${ }^{47,51,52,46}$, we observed dramatically increased Reg $3 \beta$ expression in mouse colons, IECs, and macrophages and suppressed growth of $E$. coli when IECs were treated with melatonin. Our results suggest that induction of AMPs by melatonin is a direct cause of Gram-negative bacterial suppression. Indeed, Reg3 $\beta$ deficient mice show abnormalities in inhibiting Gram-negative bacteria such as Salmonella ${ }^{47,53}$. AMPs are always expressed, but Reg3 $\beta$ is known to be expressed by TLR activation during microbial growth or inflammation ${ }^{46,54}$. Given normal physiologic conditions, the colon does not express Reg3 $\beta$ and $\alpha$-defensin but these AMPs are induced in colitis ${ }^{55}$, suggesting that TLR4 signalling 
a

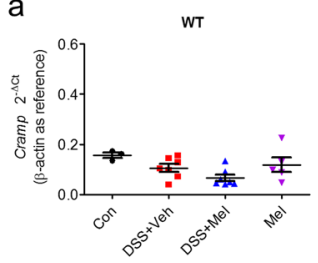

b

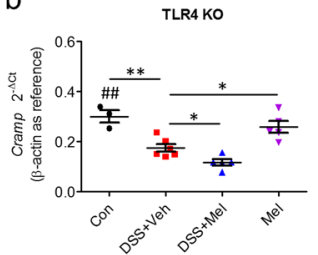

C

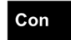

Con

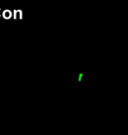

DSS+Veh
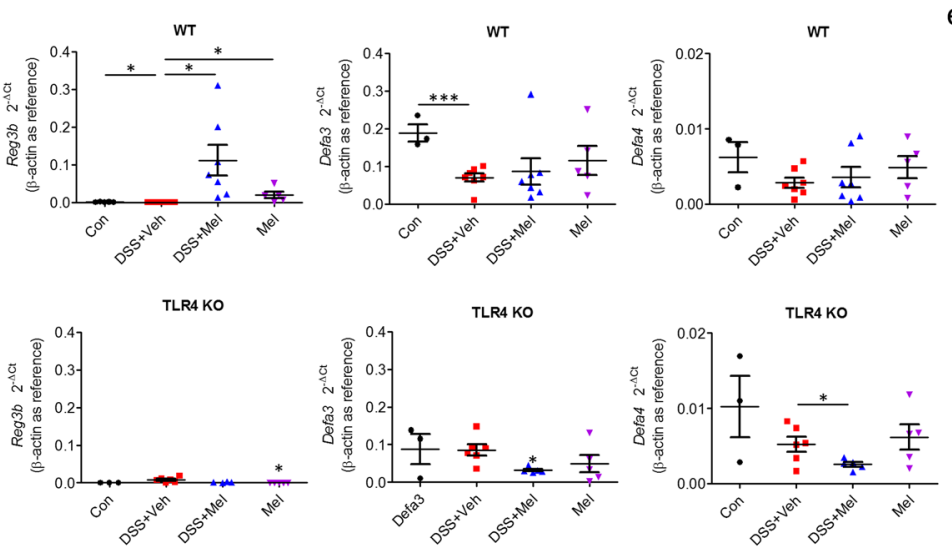

e
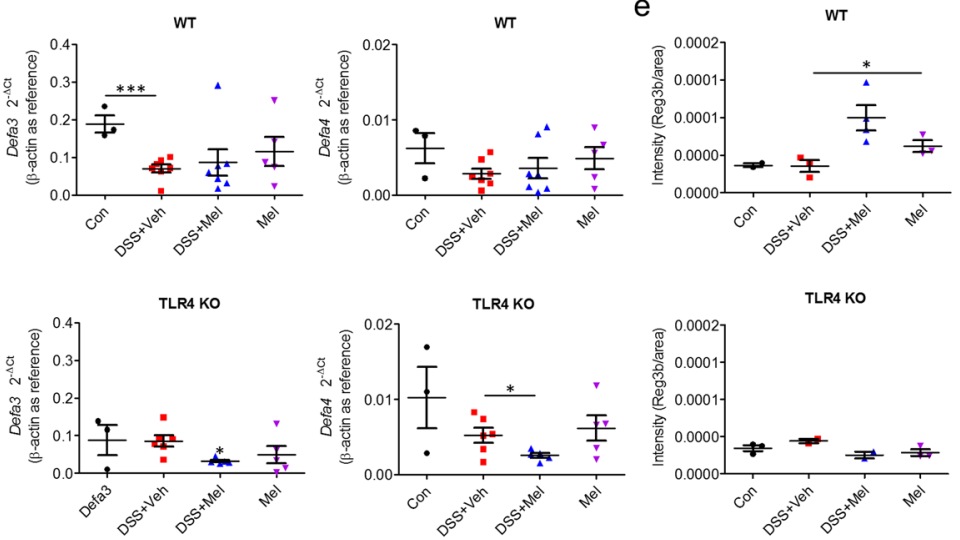

TLR4 KO
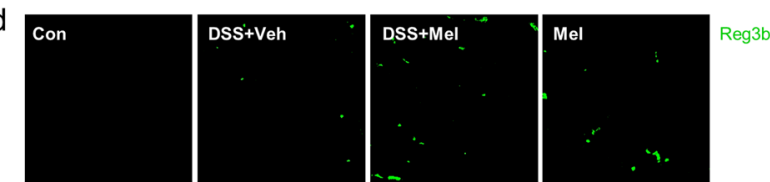

Me

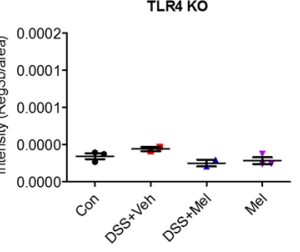

DSS'+Veh $\quad \cdot$ DSS+Mel

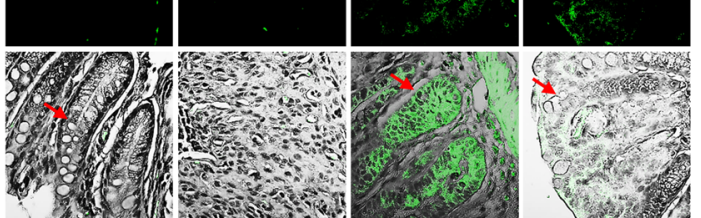

f

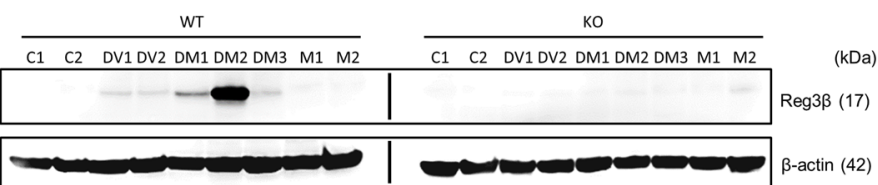

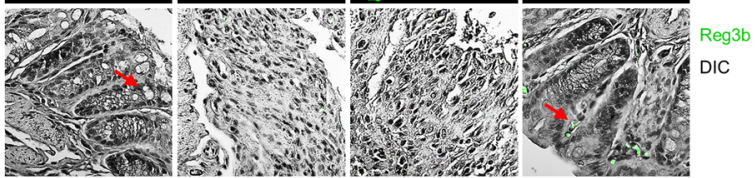

g
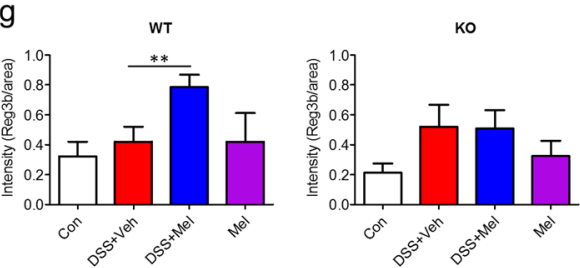

Figure 4. Melatonin promotes production of antimicrobial peptides through TLR4 signal pathway. (a,b) Gene expression of antimicrobial peptides in colon tissues. mRNA levels of Cramp, Reg3b, Defa3, and Defa4 in WT (a) and TLR4 KO (b) mice were evaluated by quantitative RT-PCR. (c-e) Protein levels of Reg3 3 in colon tissues from WT and TLR4 KO mice. (c,d) Representative image of immunofluorescence stain for Reg $3 \beta$ in colons of WT (c) and TLR4 KO (d) mice. Red arrows indicate representative goblet cells. (e) Densitometric analysis of immunofluorescence stain. Data represent mean \pm S.E.M. $(n=7)$. (f) Representative image of western blot for Reg $3 \beta$ in colon lysates of WT and TLR4 KO mice. (e) Densitometric analysis of western blot. Statistical significance was assessed using Student's t-test. $* P<0.05, * * P<0.01$, $* * * P<0.005$, ${ }^{\#} P<0.01$ vs. TLR4 KO. Con or C, untreated control; DSS or D, DSS-treated; Mel or M, melatonin-treated; TLR4 KO, TLR4 knockout mice; Veh or V, vehicle-treated; WT, wild-type mice.

also plays a role in Reg3 $\beta$ regulation by melatonin. Although melatonin has been reported to inhibit TLR4/NF- $\kappa B$ signalling ${ }^{56,57}$, TLR4 signalling appears to link pathogen sensing and AMP production is thought to play protective role in melatonin function. Experiments with TLR4 KO mice and TLR4 inhibitor revealed the anti-colitic effects of melatonin on goblet cell differentiation and induction of melatonin receptor. We demonstrated previously that TLR4 signalling is required to goblet cell differentiation ${ }^{58}$.

Reg3 $\beta$ expression also was dependent on TLR4 signalling despite a contradictory study that melatonin suppressed related TLR4 signalling ${ }^{56}$. TLR4 mediates healing and proliferation of the injured intestinal epithelium ${ }^{31,40}$ and subsequently regulates intestinal barrier function and mucosal healing ${ }^{59}$. Consistently, we found increased wound healing of IECs with melatonin, and these effects require TLR4 signalling and are mediated through macrophages. We also observed synergetic expression of mucin, antimicrobial peptides, and melatonin receptors after melatonin treatment with LPS, which is likely due to increased expression of TLR4 by LPS. Our results are also consistent with those of a previous study that TLR4 KO mice are more susceptible to colitis than WT mice ${ }^{60,61}$. Nevertheless, there may be other pathways for melatonin function than TLR4, and further mechanical study is needed to explain these contradictory results of the interaction between melatonin and TLR4 signalling.

Although further study such as conventional knock-out system is also required to confirm the exact mechanisms of AMP induction by melatonin and to exclude many confounding factors, this is the first study to explain how melatonin regulates the microbiota in colitis and to show a link between melatonin and microbiota. Additionally, we showed that bacterial sensing through TLR4, mucin, and Reg3 $\beta$ production by goblet cells was involved in the anti-colitic effects of melatonin, suggesting that melatonin may be useful in microbiota control and therapeutics for IBD. 

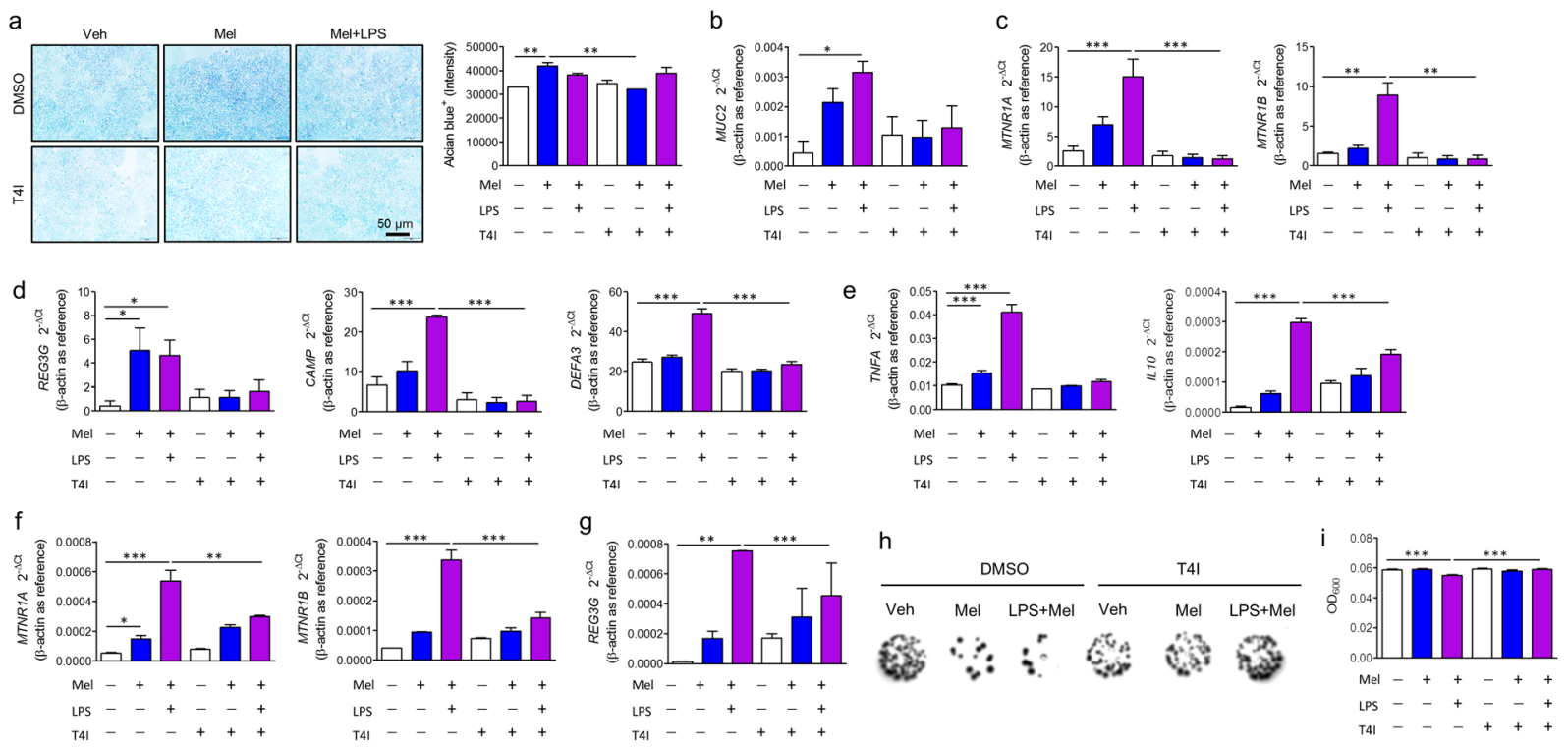

Figure 5. Melatonin controls Gram-negative bacteria by goblet cell differentiation and antimicrobial peptide production through TLR4 signal pathway. (a-d) HT-29 cells were treated with vehicle (Veh) or melatonin (Mel) with or without TLR4 inhibitor (T4I) for $48 \mathrm{~h}$. (a) Representative image of Alcian blue stain (left) and densitometry analysis (right). (b-d) Gene expressions of melatonin receptor, mucin, and antimicrobial peptides in intestinal epithelial cells (IECs). mRNA levels of mucin 2 (MUC2) (b), melatonin receptor (MTNR1A, $M T N R 1 B)(\mathbf{c})$, and antimicrobial peptides $(R E G 3 B, C A M P, D E F A 3)$ (d) were evaluated by quantitative RTPCR. Data represent means \pm S.E.M. of 3 independent experiments. (e-g) The differentiated THP-1 cells were treated with vehicle or melatonin with or without TLR4 inhibitor for $4 \mathrm{~h}$. mRNA levels of cytokines $(T N F A, I L 10)(\mathbf{e})$, melatonin receptor $(M T N R 1 A, M T N R 1 B)(\mathbf{f})$, and antimicrobial peptides $(R E G 3 B)(\mathbf{g})$ were evaluated by quantitative RT-PCR. (h,i) Antimicrobial activity of melatonin-treated cells. Escherichia coli $\left(\mathrm{OD}_{600}=0.5\right)$ grown in LB broth were treated with culture media from HT-29 cells, incubated for $48 \mathrm{~h}$, diluted at $1 / 10^{5}$, and seeded on agar plate. (h) Representative image of colonies on agar plate. Diluted bacterial cultures in the presence of HT-29 culture media were spot-inoculated onto EMB agar plate and captured in Gel document system. (i) Optical density $\left(\mathrm{OD}_{600}\right)$ of $E$. coli culture. Data represent mean \pm S.E.M. of 4 independent experiments. Statistical significance was assessed using one-way ANOVA followed by Dunnett post-test. $* P<0.05, * * P<0.01, * * * P<0.005,{ }^{*} P<0.05$ vs. DMSO, ${ }^{\# \#} P<0.01$ vs. DMSO, ${ }^{\# \#} P<0.005$ vs. DMSO. DMSO, dimethyl sulfoxide-treated; LPS, lipopolysaccharide-treated, Mel, melatonin-treated; T4I, CLI-095-treated; Veh, vehicle-treated.

a

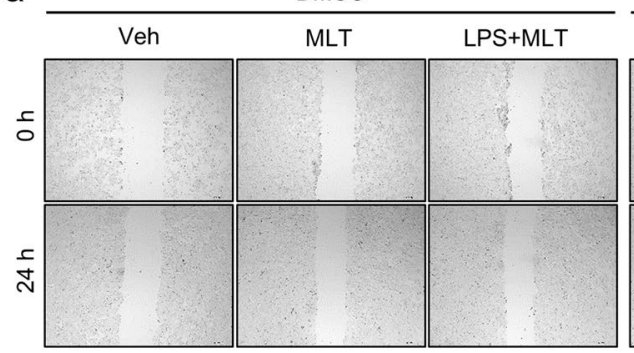

T4I

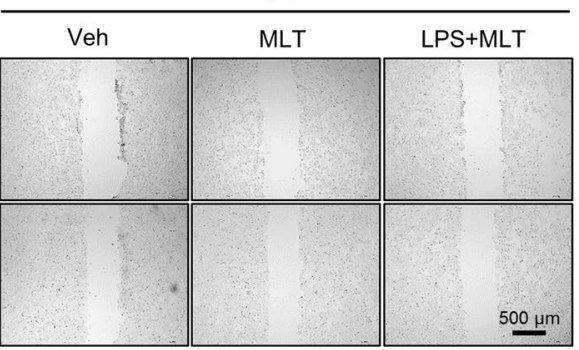

b

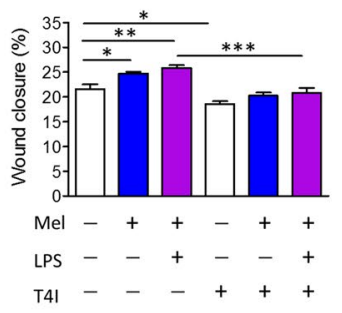

Figure 6. Melatonin improves wound healing of intestinal epithelial cells through TLR4 signal pathway. (a) Representative image of wound healing assay. (b) Wound closure rate. Wound closure rate was assessed by measuring wound area using Image J software. Data represent mean \pm S.E.M. of 3 independent experiments. Statistical significance was assessed using one-way ANOVA followed by Dunnett post-test. $* P<0.05$, ${ }^{* *} P<0.01,{ }^{\#} P<0.01$ vs. DMSO. DMSO, dimethyl sulfoxide-treated; LPS, lipopolysaccharide-treated, Mel, melatonin-treated; T4I, TLR4 inhibitor-treated; Veh, vehicle-treated.

\section{Methods}

Mouse colitis model. TLR4-deficient BALB/c mice were provided by the Korea Research Institute of Bioscience and Biotechnology (Daejon, South Korea) and were bred as previously described ${ }^{58}$. To induce acute colitis, 8-9-week-old male BALB/c mice and TLR4 knockout mice were fed 2.5\% (w/v) DSS (MW 36,000-50,000, MP Biomedicals, Solon, OH, USA) in their drinking water for 6 days. The first day of DSS administration was designated 
day 0; drinking water with DSS was replaced with normal drinking water on day 6. Mice were randomly divided into four groups: control, melatonin, DSS + vehicle, and DSS + melatonin. Control and melatonin group mice were administered only drinking water. Melatonin and DSS + melatonin groups received melatonin ( $10 \mathrm{mg} / \mathrm{kg} / \mathrm{day})$ by i.p. from days 1 to 8, while Control and DSS + vehicle groups were treated with $0.25 \% \mathrm{EtOH} / \mathrm{PBS}$ i.p based on our previous studies using DSS-induced colitis to detect rapid response $\mathrm{e}^{8,13,62}$. All mice were sacrificed on day 9, and colon length was measured. Colons and cecal contents were collected for analysis. All mice were monitored daily for body weight loss, stool consistency, and rectal bleeding. Disease activity index (DAI) scores were evaluated before sacrifice. DAI score was calculated based on the following parameters: body weight loss $(0$, none; $1,1-5 \% ; 2$, $5-10 \% ; 3,10-20 \% ; 4,>20 \%)$, rectal bleeding ( 0 , absence; 1 and 2, light bleeding; 3, bleeding), and stool consistency ( 0 , negative; 1 and 2 , loose; 3 , diarrhoea). DAI scores were expressed as the average of these three parameters. All animals were kept under standard conditions at $21-22^{\circ} \mathrm{C}$ under a $12-\mathrm{h}$ light/dark cycle. A longitudinal incision was made along the entire colon, and all stool was eliminated by washing with PBS. Parts of the proximal colon were cut into 3 pieces for PAS staining and RNA isolation. All experiments using animals were reviewed and approved by the Institutional Animal Care and Use Committee (IACUC) of Yonsei University Severance Hospital, Seoul, Korea (IACUC Approval No: 2015-0407) and were conducted in accordance with the guidelines of the IACUC.

Periodic acid-Schiff and Alcian blue staining, immunostaining, and histology. Four- $\mu$ m-thick paraffin sections of the distal colon of each animal or of cells were stained with PAS stain or Alcian blue, respectively, according to standard procedures to evaluate the histopathology and goblet cells. Immunohistochemistry was performed using anti-mouse MTNR1A (1:500; Abcam, Cambridge, MA, USA), as previously described ${ }^{58,63}$. After incubation with anti-mouse Reg3 3 (1:200, R\&D Systems, Minneapolis, MN, USA), sections were incubated with Alexa Fluor-488-conjugated secondary antibodies (Thermo Fisher Scientific, San Jose, CA, USA) for immunofluorescence stains. All images were obtained by confocal microscopy (Carl Zeiss LSM 700, Prenzlauer, Berlin, Germany), and staining intensity was determined using Image J software (NIH, Bethesda, MD, USA). Goblet cell staining was scored from 0 to 5 (5, minimal, $<10 \%$; 4, minimal, $<20 \%$; 3, mild, $21-30 \%$; , moderate, 31-40\%; 1, moderate, $41-50 \%$; 0 , marked, $>50 \%$ ).

Cell culture and treatment. HT-29 (Korea Cell Line Bank, Seoul, South Korea) and THP-1 (ATCC, Manassas, VA, USA) cell lines were maintained in $10 \%$ foetal bovine serum-supplemented RPMI medium with or without antibiotics at $37^{\circ} \mathrm{C}$ in a humidified atmosphere of $5 \% \mathrm{CO}_{2}$ with or without melatonin $(200 \mu \mathrm{M}$, Sigma-Aldrich, St. Louis, MO, USA), lipopolysaccharides (LPS, $200 \mathrm{ng} / \mathrm{mL}$, Sigma-Aldrich, St. Louis, MO, USA), and TLR4 inhibitor CLI-095 (1 $\mu \mathrm{g} / \mathrm{mL}$, InvivoGen, San Diego, CA, USA) for the indicated times. THP-1 cells were treated with a final $100 \mathrm{nM}$ of phorbol 12-myristate 13-acetate (PMA, Sigma-Aldrich) to differentiate into macrophage-like cells.

The indigenous E. coli strain previously isolated from mouse ${ }^{64}$ was kindly provided by Yoon. Bacterial culture incubated with HT-29 cell culture media was plated onto nutrient agar or EMB agar or cultured in nutrient broth at $37^{\circ} \mathrm{C}$ for $48 \mathrm{~h}$ under static culture conditions. E. coli colony images were captured by a Gel document system (MINIBIS PRO, Jerusalem, Israel), and the optical density $\left(\mathrm{OD}_{600}\right)$ of $E$. coli culture was measured using ELISA reader VERSA Max (Molecular Devices, Sunnyvale, CA, USA) and analysed using SoftMax Pro ver.6.3 (Molecular Devices).

Real-time quantitative reverse-transcription polymerase chain reaction (RT-PCR). Total RNA was prepared from colon tissues and cells using a Ribospin kit (Geneall, Seoul, Korea) and TRIzol Reagent (Life Technologies, Carlsbad, CA, USA), respectively, as per the manufacturer's instructions. RNA was reverse-transcribed using the High Capacity cDNA Reverse Transcription Kit (Applied Biosystems, Foster City, CA). The mRNA expression levels were quantified using a StepOne Plus real-time PCR system (Applied Biosystems) and SYBR Green Master Mix (Applied Biosystems). Thermal cycles were: 45 cycles of $95^{\circ} \mathrm{C}$ for $30 \mathrm{~s}$, $60-63{ }^{\circ} \mathrm{C}$ for $30 \mathrm{~s}$, and $72^{\circ} \mathrm{C}$ for $40 \mathrm{~s}$. All PCRs were run in duplicate. The results were normalized to expression of the $\beta$-actin gene, and the relative expression of each target mRNA was calculated as $2^{-\Delta \mathrm{Ct}}$. Results were normalized to expression of $\beta$-actin and were calculated by the equation $2^{-\Delta \Delta \mathrm{Ct}}$. Primer sequences were synthesized by Macrogen (Seoul, Korea). PCR primers are listed in Table S1.

Metagenome analysis of microbiome. Bacterial genomic DNA was obtained from faeces using a FastDNA ${ }^{\mathrm{TM}}$ SPIN for Soil Kit (MP Biomedicals, Santa Ana, CA, USA) according to the manufacturer's instructions. Microbiome detection using bar-coded primers flanking the V3-V4 region of the 16S rRNA gene was completed by ChunLab Inc. (Seoul, Korea). Operational taxonomic units (OTUs) were identified based on 16S rRNA sequence data using the EzTaxon-e database (http://eztaxon-e.ezbiocloud. net). Principal coordinate analysis (PCoA) of gut microbiome composition was assessed by Jensen-Shannon. OTU richness (abundance-based coverage estimator (ACE) and Chaol indices) and diversity (Shannon index and Simpson index) were analysed for alpha diversity analysis. Bacterial community abundance and composition were analysed using CLcommunity software (Chunlab Inc.).

Western blot analysis. Colon tissues were lysis harvested on ice by washing twice with cold PBS, scraping, and resuspending. The protein concentration of colon lysate was determined using the BCA assay with bovine serum albumin. Protein samples prepared by mixing SDS sample buffer were run on NuPAGE $4-12 \%$ gradient Bis-Tris and electrotransferred to a polyvinylidene difluoride membrane (Thermo Fisher Scientific) as in a previous study.64 Blots were blocked with $5 \%(\mathrm{w} / \mathrm{v})$ skim milk in Tris-buffered saline solution containing $0.1 \%$ Tween 20 (Pierce, Appleton, WI, USA) and incubated overnight at $4{ }^{\circ} \mathrm{C}$ with antibodies against $\operatorname{Reg} 3 \beta$ (1:1000, $\mathrm{R} \& \mathrm{D}$ Systems) and $\beta$-actin (Sigma). Blots were additionally incubated with secondary antibodies conjugated with horseradish peroxidase for 1 hour at room temperature and visualized with ELC Prime detection reagent and the LAS 4000 Mini apparatus (GE Healthcare, Piscataway, NJ, USA). 
Statistical analysis. GraphPad Software (La Jolla, CA, USA) was used for statistical analyses. The significance of differences between conditions was assessed using Student's $t$-test or one-way analysis of variance (ANOVA). $P$ values $<0.05$ were considered significant.

Received: 20 September 2019; Accepted: 14 January 2020; Published online: 10 February 2020

\section{References}

1. Abraham, C. \& Cho, J. H. Inflammatory bowel disease. N. Engl. J. Med. 361, 2066-2078 (2009).

2. Bassotti, G. et al. Gastrointestinal motility disorders in inflammatory bowel diseases. World J. Gastroenterol. 20, 37-44 (2014).

3. Lugering, A., Lebiedz, P., Koch, S. \& Kucharzik, T. Apoptosis as a therapeutic tool in IBD? Ann. N. Y. Acad. Sci. 1072, 62-77 (2006).

4. Baumgart, D. C. \& Sandborn, W. J. Inflammatory bowel disease: clinical aspects and established and evolving therapies. Lancet 369, 1641-1657 (2007).

5. Paramsothy, S. et al. Specific Bacteria and Metabolites Associated With Response to Fecal Microbiota Transplantation in Patients With Ulcerative Colitis. Gastroenterology 156, 1440-1454 e1442 (2019).

6. Reiter, R. J. et al. Melatonin as an antioxidant: under promises but over delivers. J. Pineal Res. 61, 253-278 (2016).

7. Calvo, J. R., Gonzalez-Yanes, C. \& Maldonado, M. D. The role of melatonin in the cells of the innate immunity: a review. J. Pineal Res. 55, 103-120 (2013).

8. Park, Y. S. et al. Melatonin improves experimental colitis with sleep deprivation. Int. J. Mol. Med. 35, 979-986 (2015).

9. Esteban-Zubero, E. et al. Melatonin's role as a co-adjuvant treatment in colonic diseases: A review. Life Sci. 170, 72-81 (2017).

10. Gao, T. et al. Role of melatonin in sleep deprivation-induced intestinal barrier dysfunction in mice. J. Pineal Res. 67, e12574 (2019).

11. Thor, P. J. et al. Melatonin and serotonin effects on gastrointestinal motility. J. Physiol. Pharmacol. 58(Suppl 6), 97-103 (2007).

12. Siah, K. T., Wong, R. K. \& Ho, K. Y. Melatonin for the treatment of irritable bowel syndrome. World J. Gastroenterol. 20, 2492-2498 (2014).

13. Chung, S. H. et al. Melatonin attenuates dextran sodium sulfate induced colitis with sleep deprivation: possible mechanism by microarray analysis. Dig. Dis. Sci. 59, 1134-1141 (2014).

14. Guven, A. et al. Melatonin ameliorates necrotizing enterocolitis in a neonatal rat model. J. Pediatr. Surg. 46, 2101-2107 (2011).

15. Stebelova, K., Herichova, I. \& Zeman, M. Diabetes induces changes in melatonin concentrations in peripheral tissues of rat. Neuro Endocrinol. Lett. 28, 159-165 (2007).

16. Bubenik, G. A. Thirty four years since the discovery of gastrointestinal melatonin. J. Physiol. Pharmacol. 59(Suppl 2), 33-51 (2008).

17. Ren, W. et al. Melatonin alleviates weanling stress in mice: Involvement of intestinal microbiota. J Pineal Res 64 (2018).

18. Meylan, E., Tschopp, J. \& Karin, M. Intracellular pattern recognition receptors in the host response. Nat. 442, 39-44 (2006).

19. Kordjazy, N. et al. Role of toll-like receptors in inflammatory bowel disease. Pharmacol. Res. 129, 204-215 (2018).

20. O'Brien, G. C., Wang, J. H. \& Redmond, H. P. Bacterial lipoprotein induces resistance to Gram-negative sepsis in TLR4-deficient mice via enhanced bacterial clearance. J. Immunol. 174, 1020-1026 (2005).

21. Hoshino, K. et al. Cutting edge: Toll-like receptor 4 (TLR4)-deficient mice are hyporesponsive to lipopolysaccharide: evidence for TLR4 as the Lps gene product. J. Immunol. 162, 3749-3752 (1999).

22. Masana, M. I. \& Dubocovich, M. L. Melatonin receptor signaling: finding the path through the dark. Sci. STKE 2001, pe39 (2001).

23. Stebelova, K. et al. Immunohistochemical definition of MT(2) receptors and melatonin in the gastrointestinal tissues of rat. Acta Histochem. 112, 26-33 (2010).

24. Sotak, M., Mrnka, L. \& Pacha, J. Heterogeneous expression of melatonin receptor MT1 mRNA in the rat intestine under control and fasting conditions. J. Pineal Res. 41, 183-188 (2006).

25. Soderquist, F., Hellstrom, P. M. \& Cunningham, J. L. Human gastroenteropancreatic expression of melatonin and its receptors MT1 and MT2. PLoS One 10, e0120195 (2015).

26. Geirnaert, A. et al. Butyricicoccus pullicaecorum, a butyrate producer with probiotic potential, is intrinsically tolerant to stomach and small intestine conditions. Anaerobe 30, 70-74 (2014).

27. Geirnaert, A. et al. Butyrate-producing bacteria supplemented in vitro to Crohn's disease patient microbiota increased butyrate production and enhanced intestinal epithelial barrier integrity. Sci. Rep. 7, 11450 (2017).

28. Xu, P. et al. Melatonin prevents obesity through modulation of gut microbiota in mice. J Pineal Res 62 (2017).

29. Burger-van Paassen, N. et al. Mucin Muc2 deficiency and weaning influences the expression of the innate defense genes Reg3beta, Reg3gamma and angiogenin-4. PLoS One 7, e38798 (2012).

30. Xia, Y. et al. Melatonin in macrophage biology: Current understanding and future perspectives. J. Pineal Res. 66, e12547 (2019).

31. Fukata, M. \& Abreu, M. T. TLR4 signalling in the intestine in health and disease. Biochem. Soc. Trans. 35, 1473-1478 (2007).

32. Tan, D. X. et al. Melatonin as a Potent and Inducible Endogenous Antioxidant: Synthesis and Metabolism. Molecules 20, 18886-18906 (2015).

33. MacEachern, S. J. et al. Alterations in melatonin and 5-HT signalling in the colonic mucosa of mice with dextran-sodium sulfateinduced colitis. Br. J. Pharmacol. 175, 1535-1547 (2018).

34. Cuzzocrea, S. et al. Melatonin reduces dinitrobenzene sulfonic acid-induced colitis. J. Pineal Res. 30, 1-12 (2001).

35. Trivedi, P. P. \& Jena, G. B. Melatonin reduces ulcerative colitis-associated local and systemic damage in mice: investigation on possible mechanisms. Dig. Dis. Sci. 58, 3460-3474 (2013).

36. Bubenik, G. A. \& Brown, G. M. Pinealectomy reduces melatonin levels in the serum but not in the gastrointestinal tract of rats. Biol. Signals 6, 40-44 (1997).

37. Sartor, R. B. Microbial influences in inflammatory bowel diseases. Gastroenterology 134, 577-594 (2008)

38. Necefli, A. et al. The effect of melatonin on TNBS-induced colitis. Dig. Dis. Sci. 51, 1538-1545 (2006).

39. Pentney, P. T. \& Bubenik, G. A. Melatonin reduces the severity of dextran-induced colitis in mice. J. Pineal Res. 19, 31-39 (1995).

40. Li, J. et al. Effect of melatonin on renewal of chicken small intestinal mucosa. Poult. Sci. 96, 2942-2949 (2017).

41. Van der Sluis, M. et al. Muc2-deficient mice spontaneously develop colitis, indicating that MUC2 is critical for colonic protection. Gastroenterology 131, 117-129 (2006).

42. Dheer, R. et al. Intestinal Epithelial Toll-Like Receptor 4 Signaling Affects Epithelial Function and Colonic Microbiota and Promotes a Risk for Transmissible Colitis. Infect. Immun. 84, 798-810 (2016).

43. Zaiss, D. M. W., Gause, W. C., Osborne, L. C. \& Artis, D. Emerging functions of amphiregulin in orchestrating immunity, inflammation, and tissue repair. Immun. 42, 216-226 (2015).

44. Ouwerkerk, J. P., de Vos, W. M. \& Belzer, C. Glycobiome: bacteria and mucus at the epithelial interface. Best. Pract. Res. Clin. Gastroenterol. 27, 25-38 (2013).

45. Johansson, M. E. et al. Normalization of Host Intestinal Mucus Layers Requires Long-Term Microbial Colonization. Cell Host Microbe 18, 582-592 (2015).

46. Vaishnava, S. et al. Paneth cells directly sense gut commensals and maintain homeostasis at the intestinal host-microbial interface. Proc. Natl Acad. Sci. USA 105, 20858-20863 (2008). 
47. van Ampting, M. T. et al. Intestinally secreted C-type lectin Reg3b attenuates salmonellosis but not listeriosis in mice. Infect. Immun. 80, 1115-1120 (2012).

48. Loonen, L. M. et al. REG3gamma-deficient mice have altered mucus distribution and increased mucosal inflammatory responses to the microbiota and enteric pathogens in the ileum. Mucosal Immunol. 7, 939-947 (2014).

49. Chow, J. C. et al. Toll-like receptor-4 mediates lipopolysaccharide-induced signal transduction. J. Biol. Chem. 274, 10689-10692 (1999).

50. Sodhi, C. P. et al. Intestinal epithelial Toll-like receptor 4 regulates goblet cell development and is required for necrotizing enterocolitis in mice. Gastroenterology 143, 708-718 e705 (2012).

51. Miki, T., Holst, O. \& Hardt, W. D. The bactericidal activity of the C-type lectin RegIIIbeta against Gram-negative bacteria involves binding to lipid A. J. Biol. Chem. 287, 34844-34855 (2012).

52. Stelter, C. et al. Salmonella-induced mucosal lectin RegIIIbeta kills competing gut microbiota. PLoS One 6, e20749 (2011).

53. Wang, L. et al. Intestinal REG3 Lectins Protect against Alcoholic Steatohepatitis by Reducing Mucosa-Associated Microbiota and Preventing Bacterial Translocation. Cell Host Microbe 19, 227-239 (2016).

54. Brandl, K. et al. MyD88-mediated signals induce the bactericidal lectin RegIII gamma and protect mice against intestinal Listeria monocytogenes infection. J. Exp. Med. 204, 1891-1900 (2007).

55. Cunliffe, R. N. et al. Human defensin 5 is stored in precursor form in normal Paneth cells and is expressed by some villous epithelial cells and by metaplastic Paneth cells in the colon in inflammatory bowel disease. Gut 48, 176-185 (2001).

56. Chamanara, M. et al. Melatonin ameliorates TNBS-induced colitis in rats through the melatonin receptors: involvement of TLR4/ MyD88/NF-kappaB signalling pathway. Inflammopharmacology 27, 361-371 (2019).

57. Renn, T. Y. et al. Prophylactic supplement with melatonin successfully suppresses the pathogenesis of periodontitis through normalizing RANKL/OPG ratio and depressing the TLR4/MyD88 signaling pathway. J Pineal Res 64 (2018).

58. Seo, D. H. et al. Interleukin-33 regulates intestinal inflammation by modulating macrophages in inflammatory bowel disease. Sci. Rep. 7, 851 (2017).

59. Rakoff-Nahoum, S. et al. Recognition of commensal microflora by toll-like receptors is required for intestinal homeostasis. Cell 118, 229-241 (2004)

60. Fukata, M. et al. Toll-like receptor-4 is required for intestinal response to epithelial injury and limiting bacterial translocation in a murine model of acute colitis. Am. J. Physiol. Gastrointest. Liver Physiol 288, G1055-1065 (2005).

61. Tamtaji, O. R. et al. Melatonin, a toll-like receptor inhibitor: Current status and future perspectives. J. Cell Physiol. 234, 7788-7795 (2019).

62. Kim, T. K. et al. Melatonin modulates adiponectin expression on murine colitis with sleep deprivation. World J. Gastroenterol. 22, 7559-7568 (2016).

63. Kim, S. W. et al. Identification of genetic susceptibility loci for intestinal Behcet's disease. Sci. Rep. 7, 39850 (2017).

64. Yoon, M. Y. et al. A single gene of a commensal microbe affects host susceptibility to enteric infection. Nat. Commun. 7, 11606 (2016).

\section{Acknowledgements}

This work was supported by the National Research Foundation of Korea (NRF) grant funded by the Korea Government, Ministry of Science and ICT government (MSIT) (No. NRF-2016R1A2B1016426).

\section{Author contributions}

All authors were involved in drafting the article or critically revising it for important intellectual content, and all authors approved the final version for publication. Dr. Y. S.P. had full access to all data in the study and takes responsibility for data integrity and accuracy of the data analysis. Study concept and design: Y. S.P., J.H.C. and S.W.K. Acquisition of data: S.W.K., S.K. and M.S. Analysis and interpretation of data: S.W.K., S.K., M. S., Y. S.P. and J.H.C.

\section{Competing interests}

The authors declare no competing interests.

Additional information

Supplementary information is available for this paper at https://doi.org/10.1038/s41598-020-59314-7.

Correspondence and requests for materials should be addressed to Y.S.P.

Reprints and permissions information is available at www.nature.com/reprints.

Publisher's note Springer Nature remains neutral with regard to jurisdictional claims in published maps and institutional affiliations.

(c) (i) Open Access This article is licensed under a Creative Commons Attribution 4.0 International (c) License, which permits use, sharing, adaptation, distribution and reproduction in any medium or format, as long as you give appropriate credit to the original author(s) and the source, provide a link to the Creative Commons license, and indicate if changes were made. The images or other third party material in this article are included in the article's Creative Commons license, unless indicated otherwise in a credit line to the material. If material is not included in the article's Creative Commons license and your intended use is not permitted by statutory regulation or exceeds the permitted use, you will need to obtain permission directly from the copyright holder. To view a copy of this license, visit http://creativecommons.org/licenses/by/4.0/.

(c) The Author(s) 2020 